Paulo Conti, até hoje um dos maiores nomes internacionais na área de dor orofacial e disfunção temporomandibular. Sinto-me orgulhoso por ter, na qualidade de seu professor, no terceiro ano da graduação, percebido seu interesse pela área de oclusão e iniciado o período de convivência que resultou em uma nítida superação do mestre pelo discípulo. Ao terminar seu curso de graduação da Faculdade de Odontologia de Bauru, aquele discreto aluno surpreendeu a todos, quando a média de suas notas ao longo de todo o curso foi a maior da turma, conferindo-lhe o prêmio de melhor aluno da USP Bauru na sua formatura. Logo iniciou seu treinamento com um periodo de residência no centrinho Bauru, que resultou no início de sua maravilhosa produção cientifica. Ao meu retorno de um treinamento nos EUA na área de disfunção em 1991 iniciamos nossas atividades conjuntas em pesquisa e ensino da dor. A partir daí a carreira do professor Paulo Conti caminhou à velocidade da luz. Seu doutorado brilhante estimulou-o a procurar um centro mundial de conhecimento, a Universidade de Nova Jersey, onde tendo chegado como um estudante qualquer, de lá retornou como um colega e colaborador do maior nome, na ocasião, da dor orofacial em todo o mundo, o professor Richard Pertes, autor do livro mais importante na área para os dentistas. Em sua volta, Conti trouxe consigo os sinais de maturidade científica e liderança. Iniciou na Faculdade de Odontologia de Bauru um programa denso de pesquisas que consolidou a clareza da separação científica entre os assuntos de oclusão e de dor. Suas publicações desde então o credenciam como uma das maiores expressões acadêmicas da Faculdade de Bauru. Decidiu prestar o exame de ingresso nos EUA, para tornar-se membro da academia norte-americana de dor orofacial - uma exposição delicada em nível pessoal, jamais tentada antes por um brasileiro - porém que resultou em uma brilhante aprovação e abriu as portas para os nossos grupos de pesquisa partilharem, no mundo científico do mais alto nível, o espaço nas publicações internacionais. O professor Paulo Conti coordena hoje um dos programas de maior renome internacional em pesquisa na área de dor e disfunção temporomandibulares. É presença constante no cenário internacional e eleva o nome da Odontologia brasileira, levando inéditas informações geradas daqui aos mais diferentes pontos do planeta. No próximo dia 29 deste mês, por exemplo, estará em Las Vegas apresentando à academia norte-americana os últimos resultados de pesquisa na área de dor desenvolvidas por sua equipe. Paulo Conti é uma expressão internacional e orgulhosamente, para nós de Bauru, um discipulo que superou os mestres.

Professor Doutor Carlos dos Reis Pereira de Araújo

\section{Paulo Conti}

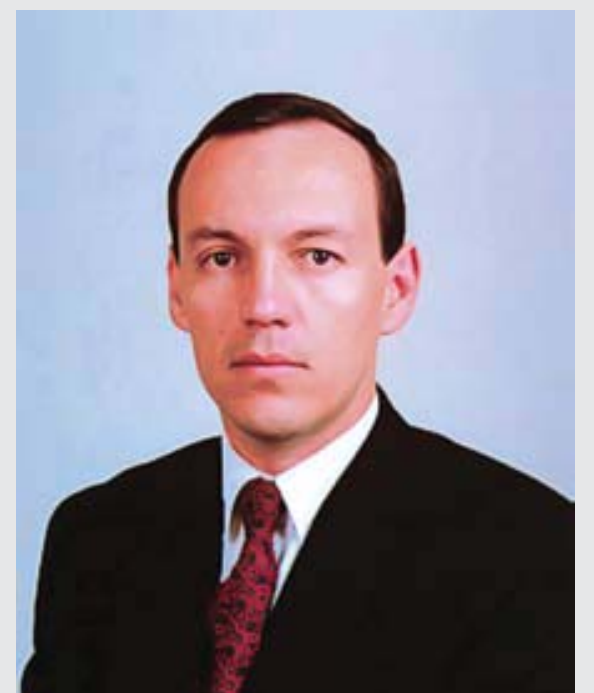

Professor Associado do Departamento de Prótese da Faculdade de Odontologia de Bauru, da Universidade de São Paulo.

Mestrado e Doutorado em Reabilitação Oral pela FOB-USP.

Pós-Doutorado em Disfunções Temporomandibulares e Dores Orofaciais pela University of Medicine and Dentistry of New Jersey, USA. "Diplomate" American Board of Orofacial Pain.

Coordenador do Curso de Especialização em Prótese Dentária da FOB-USP.

Presidente da Comissão de Biblioteca e Documentação da FOB-USP.

- Coordenador do Programa de Pós-Graduação em Reabilitação Oral da FOB-USP.

Infelizmente, no entanto, trabalhos de pesquisa nesta área geralmente são longitudinais, de longa duração e dependem da participação de pacientes específicos para que os mesmos tenham validade e possam ter um espaço em publicações internacionais. Isso faz com que o número de publicações de trabalhos de nível nessa área tão carente de informações concretas e confiáveis também seja pequeno, quando comparado com outras áreas da Odontologia.

Apesar de relativamente recente em nosso país, nossa especialidade tem conseguido ocupar papel de destaque em publicações nacionais, com o objetivo de divulgá-la para a classe odon- 
tológica. Obviamente, encontramos uma certa resistência de profissionais ainda enraizados em conceitos meramente morfológicos relacionados à etiologia e tratamento das desordens da ATM, como a má oclusão dentária, por exemplo. Isso ainda resulta em tratamentos onerosos, desnecessários e que põem em questão a credibilidade de nossa especialidade. A transferência da ciência, ou seja, a aplicação clínica das evidências científicas continua sendo um grande desafio para todos nós, envolvidos com o ensino de graduação e pós-graduação no campo das Disfunções Temporomandibulares e Dores Orofaciais.

Talvez o melhor caminho para solucionar estes problemas seja um controle mais rígido do conteúdo dos cursos de especialização na área, aliado à criação de disciplinas nos cursos de graduação de Odontologia. Campanhas de conscientização da população também poderiam ter um impacto positivo para a especialidade, pois poucas pessoas sabem que existem profissionais especificamente treinados para diagnosticar e tratar tais problemas.

2) DTM e Dor Orofacial, dois temas distintos muito extensos em conhecimento e capacitação profissional, como atuar em ambas? Para qual nível de atuação, em cada uma delas, o cirurgião-dentista está amplamente habilitado? Sérgio Guimarães

Manifestações de sintomas de Disfunção Temporomandibular (DTM) são consideradas dores orofaciais de origem músculo-esquelética e constituem a grande maioria das queixas apresentadas por pacientes de dor facial, excluindo-se, obviamente, aquelas dores de origem dentária.

Creio que o reconhecimento do problema e encaminhamento para especialistas deva ser a rotina daqueles não capacitados para tal. Há condições, em que se requer somente orientações relativas a cuidados caseiros e mudança de hábitos, que um clínico bem informado poderia ser útil para a melhora dos sintomas.

Indivíduos com dores faciais crônicas, refratá- rios a terapias convencionais, apresentando dores do tipo neuropática, e co-morbidade com cefaléias primárias deveriam sempre serem tratados por um grupo especializado que inclui dentistas, médicos, fisioterapeutas, psicólogos, etc. Em um trabalho ${ }^{2}$ de pesquisa realizado em nosso serviço de urgência odontológica do Campus da USP, em Bauru, encontramos prevalência de sintomas de DTM em $60 \%$ da população, sendo que, destes, 54\% também apresentavam sintomas de cefaléia primária tensional ou de migrânea. Este fato reitera a necessidade de se trabalhar em grupo para um melhor atendimento do paciente.

\section{3) Existem fármacos que influenciam o bruxismo noturno? Se afirmativo, quais são e como agem? Eleutério Araújo Martins}

O relacionamento e a participação de fármacos na presença e severidade do bruxismo noturno é bastante discutido na literatura. Apesar de estudados em alguns trabalhos de pesquisa, nenhuma conclusão definitiva pôde ser tirada da maioria deles.

Winocur et al. ${ }^{3}$ realizaram uma extensa revisão sobre a participação de todos os tipos de fármacos no bruxismo noturno e concluíram que ainda não há estudos controlados que possam ser conclusivos nesse tópico.

Parece óbvio que algumas drogas usadas no controle das dores crônicas e de quadros depressivos alteram de certa forma o sono e a concentração de neurotransmissores no SNC. Este fato poderia levar a alterações na qualidade e arquitetura do sono, com algum envolvimento do bruxismo.

Alguns trabalhos ${ }^{4,5}$ sugeriram que a classe dos antidepressivos do tipo Inibidores Seletivos da Recaptação da Serotonina (ISRS) poderiam estimular ou potencializar desordens de movimentos, incluindo-se o bruxismo pelo aumento na concentração de serotonina e interações com o sistema dopaminérgico. A insônia é também um efeito colateral freqüente de pacientes submetidos a terapia com estes fármacos. Fazem parte desta classe fármacos muito utilizados em nossos dias, como a 
fluoxetina e a paroxetina, para tratamento da depressão. Tal fato deve-se aos efeitos colaterais bem mais brandos, quando comparados com os antidepressivos "tradicionais". Assim, o clínico deve ficar atento para o possível surgimento repentino de sinais e sintomas de bruxismo nos seus pacientes, como fratura de restaurações e coroas de porcelana, cansaço muscular matinal, dores musculares, etc. Este efeito excitatório destes medicamentos parece ser amenizado com o uso concomitante de beta-bloqueadores (propanolol, nadolol, etc.).

Por outro lado, outra classe de antidepressivos, os tricíclicos têm sido associados à diminuição na freqüência e severidade do hábito noturno de bruxismo. Talvez este fato esteja relacionado à grande gama de atuação destes fármacos em diferentes receptores no SNC, o que gera mais efeitos colaterais, incluindo-se a sedação e diminuição de sono REM ("rapid eye movement"). Não há, no entanto trabalhos controlados que provem esta hipótese. Dentro desta classe, encontram-se os antidepressivos da primeira geração, as aminas terciárias e secundárias, como a amitriptilina, desipramina, nortriptilina, etc. Já a venlafaxina, também bastante utilizada nos últimos anos no tratamento de depressão e dores crônicas das mais diferentes origens, parece excitar o bruxismo, assim como os neurolépticos.

\section{4) Quais os avanços mais significativos nos últimos dois anos na área da dor orofacial? Eleutério Araújo Martins}

Acredito que um melhor entendimento de mecanismos de transmissão da dor e meios de supressão intrínseca destas dores foram marcos importantes para o desenvolvimento de novas drogas específicas para o tratamento de dores crônicas e neuropáticas. A caracterização psicosocial do paciente de dor facial também tem sido de extrema valia para a formulação de terapias cognitivas comportamentais individualizadas, como parte do tratamento destes pacientes refratários aos tratamentos convencionais.
A imagenologia dinâmica do SNC também nos dará em um futuro próximo, informações muito importantes sobre ativação de determinadas áreas do cérebro em pacientes com dor, sob determinadas circunstâncias, o que pode abrir um novo campo de pesquisa para o desenvolvimento de novas terapias.

Ainda, o reconhecimento da participação da expressão de receptores centrais e periféricos na manutenção das dores orofaciais foi um passo importante para o entendimento do paciente neuropático, abrindo perspectivas para um tratamento mais eficaz.

Todos esses avanços esbarram, no entanto, em algumas dificuldades históricas. A tabela 1 ilustra esse cenário.

Tabela 1 - Dores orofaciais.

\begin{tabular}{ccc} 
Avanços & Futuro & Problemas \\
$\begin{array}{c}\text { Identificação me- } \\
\text { canismos centrais e } \\
\text { periféricos }\end{array}$ & $\begin{array}{c}\text { Drogas: agonistas } \\
\text { e antagonistas }\end{array}$ & Dores referidas \\
$\begin{array}{c}\text { Novas drogas e } \\
\text { controle } \\
\text { farmacológico }\end{array}$ & $\begin{array}{c}\text { Melhor entendi- } \\
\text { mento interpreta- } \\
\text { ção da dor }\end{array}$ & $\begin{array}{c}\text { Dor por estímulos } \\
\text { suaves }\end{array}$ \\
$\begin{array}{c}\text { Participação de fatores } \\
\text { psicosociais }\end{array}$ & $\begin{array}{c}\text { Entendimento de } \\
\text { neuroquímicos }\end{array}$ & $\begin{array}{c}\text { Sensitização } \\
\text { neuronal }\end{array}$ \\
Educação em pós- & Imagenologia & Mecanismos de dor \\
graduação & da dor & aguda para crônica \\
Terapias reversíveis & & \\
\hline
\end{tabular}

\section{5) Quais os procedimentos atualmente considerados os mais seguros no tratamento da dor orofacial? Eleutério Araújo Martins}

Todo tratamento é seguro quando o profissional foi devidamente treinado e está amplamente habilitado para executá-lo. Por isso a criação da especialidade de Disfunção Temporomandibular e Dores Orofaciais tem um papel importante. Em linhas gerais, é unânime que as terapias não invasivas e reversíveis devem sempre ser a primeira opção. 
Neste aspecto, um correto diagnóstico, baseado em anamnese, exame físico e, em alguns casos, exames complementares é de fundamental importância para um planejamento correto. Acho que diagnósticos incorretos, que levam à instituição de terapias desnecessárias, sejam o maior problema para os colegas que relatam fracassos em seus pacientes de dor facial. Muitas vezes, tratamentos considerados inócuos por muitos, como a utilização de uma placa oclusal, podem ser prejudiciais ao paciente se não forem corretamente utilizados, controlados e acompanhados. Alterações oclusais irreversíveis, devidas a uso inadequado de placas oclusais com desenhos e formas das mais variadas, são rotina em clínicas de atendimento de pacientes com dor facial.

A relação custo-benefício também deve ser discutida com o paciente. Muitos tratamentos longos e caros nem sempre resultam na melhora almejada pelo paciente. $\mathrm{O}$ ajuste oclusal por desgaste seletivo é um bom exemplo desse problema. Historicamente usado como terapia definitiva para dores músculo-esqueléticas tipo DTM (e muitas vezes até de maneira indiscriminada para dores neuropáticas e vasculares....), sua utilização tem sido questionada por revisões sistemáticas onde comprova-se sua ineficácia na melhora dos sintomas. A tabela 2 mostra alguns desses trabalhos.

Tabela 2 - Revisão sistemática ${ }^{6}$.

\begin{tabular}{ccccc}
\hline & Follow-up & $\begin{array}{c}\text { Masca- } \\
\text { ram. }\end{array}$ & Qualid. & Resultado \\
\cline { 2 - 5 } $\begin{array}{c}\text { Werndahl } \\
\text { et al. }\end{array}$ & $\begin{array}{c}6 \text { sema- } \\
\text { nas }\end{array}$ & não & 0,24 & $=$ controle \\
$\begin{array}{c}\text { Wenneberg et } \\
\text { al. 1988 }\end{array}$ & 2 meses & não & 0,40 & $\begin{array}{c}\text { = menor } \\
\text { controle }\end{array}$ \\
$\begin{array}{c}\text { Vallon et al. } \\
1991 / 5\end{array}$ & $\begin{array}{c}1,3,6 \\
\text { meses }\end{array}$ & simples & 0,57 & $\begin{array}{c}\text { controle } \\
\text { passivo }\end{array}$ \\
$\begin{array}{c}\text { Tsolka et al. } \\
1992\end{array}$ & 10 dias & duplo & 0,36 & $\begin{array}{c}\text { controle } \\
\text { placebo }\end{array}$ \\
\hline
\end{tabular}

6) Qual, a seu critério, a importância que deve ter a dor orofacial em programas de saúde pública? Eleutério Araújo Martins
Apesar da alta prevalência de algum sintoma (35\%) e de algum sinal (65\%), as dores faciais de origem músculo-esqueléticas são, em sua maioria, de intensidade branda, transitórias, auto-limitantes.

Isso faz com que a necessidade de tratamento fique restrita a uma parcela bem menor de pacientes. Os problemas são muito mais sérios em termos de saúde pública, se considerarmos aqueles que se tornam crônicos, com todas as conseqüências financeiras, perdas sociais e de qualidade de vida envolvidas nestes casos.

A co-existência de outras patologias com as dores faciais, como a fibromialgia, as cefaléias primárias, cervicalgias, quadros depressivos, faz com que este paciente torne-se um excluído de seu ambiente de convívio social, afastando-se de atividades rotineiras, inclusive profissionais.

Algumas tentativas têm sido feitas com o objetivo de melhorar o atendimento ao paciente de dor crônica em nosso país, porém sem um direcionamento específico para as dores faciais.

7) Existem dados sobre a prevalência da dor crônica na população brasileira semeIhantes aos apresentados, para outras populações, nos Congressos da EFIC em Genève (2004) e da IASP, em Sidney (2005)? Eleutério Araújo Martins

De acordo com a SBED (Sociedade Brasileira para o Estudo da Dor) não existem dados estatísticos oficiais sobre a dor no Brasil, mas a sua ocorrência tem aumentado substancialmente nos últimos anos.

A dor afeta pelo menos 30\% dos indivíduos durante algum momento da sua vida e, em 10 a 40\% deles, tem duração superior a um dia, ocasionando graves conseqüências psicossociais e econômicas. Ainda, de acordo com as publicações do grupo do Dr. Manoel Jacobsen Teixeira, do Centro de Dor do Hospital das Clínicas da USP, a incidência da dor crônica no mundo oscila entre $7 \%$ e $40 \%$ da população e, como conseqüência da mesma, cerca de 50 a 60\% têm um comprometimento significa- 
tivo na qualidade de vida.

No Brasil, 10 a $50 \%$ dos pacientes procuram clínicas gerais devido a queixas de dor, sendo que a fibromialgia, síndrome dolorosa miofascial e artralgias são mais freqüentes em mulheres. Já os homens procuram o Centro de Dor da USP mais por dores de câncer, dores decorrentes de amputações, afeccções isquêmicas, mielopatias e encefalopatias ${ }^{7}$.

Ainda, 1/3 de nossa população, quando questionada por meio de telefone, julga que a dor crônica compromete as atividades habituais e mais de $3 / 4$ acham que a dor crônica é limitante para as atividades de recreação, sociais e familiares ${ }^{8}$.

Estes dados demonstram a importância e o impacto que as dores crônicas têm no dia-a dia dos indivíduos e a necessidade de medidas sérias que possam levar o alívio destes problemas a toda população necessitada.

8) McNamara, Seligman e Okeson' fizeram uma extensa revisão sobre oclusão, tratamento ortodôntico e desordens da ATM, citaram uma baixa inter-relação entre eles, entretanto, caracterizaram 5 fatores oclusais de risco. Na sua opinião, isto só alimenta a controvérsia DTM X oclusão, ou pacientes refratários com tais características na oclusão devem tratá-las? Elcy Arruda

Este é um aspecto bastante importante a ser considerado quando se está diante de um paciente com DTM e algum dos fatores de risco citados pelo grupo da UCLA, em várias publicações.

Você tem razão, quando cita a possível confusão que esses resultados podem gerar, pois muitos dentistas podem assumir que o tratamento dos fatores de risco poderia tratar o paciente com DTM, o que não é verdade. Fator de risco oclusal é definido, neste caso, como sendo uma condição que, quando presente, requer uma capacidade adaptativa mais acurada do indivíduo, para que ocorra uma acomodação fisiológica a essa condição. Em linhas gerais, isto acontece na grande maioria da população que apresenta algum desses fatores de risco.

Quando detectada em indivíduos sintomáticos, deve ficar claro que o tratamento da condição oclusal não está indicado para melhorar a dor e disfunção, pois não se pode afirmar, no momento do diagnóstico, qual o grau de participação do fator oclusal em cada caso. Ainda, não podemos nos esquecer que muitas disfunções podem causar alterações oclusais como conseqüência, o que contra-indicaria qualquer tipo de terapia oclusal irreversivel imediata.

O tratamento desses fatores de risco em indivíduos assintomáticos jovens, no entanto, pode fazer com que o sistema desenvolva-se de maneira mais adequada. Não se pode afirmar, no entanto, que isso seria prevenção de DTM, pois todos sabemos o caráter multifatorial da etiologia das mesmas.

A tabela 3 explica essas dúvidas em relação à necessidade ou não de intervenção.

Tabela 3 - Dúvidas.

\section{Devo tratá-los preventivamente?}

Não, com o único objetivo de prevenir DTM. Porém, o tratamento de alguns fatores pode diminuir a necessidade da existência de uma capacidade adaptativa apropriada em alguns indivíduos. Isto pode ser um fator positivo no futuro, se o indivíduo vier a ter outro fator contribuinte, como hábitos parafuncionais, macrotraumas, etc.

\footnotetext{
A terapia corretiva dos fatores de risco leva à "cura" do paciente?

Não, processos de desarranjos internos da ATM não são revertidos com terapias oclusais em adultos sintomáticos. Processos de sensibilização central podem manter os sintomas, independente da terapia periférica ou de correção dos fatores de risco por meio de Ortodontia, prótese ou ajuste oclusal.
}

\section{9) O senhor acha que a toxina botulínica já} é uma realidade para os profissionais que se propõem a tratar DTM e DOF? Elcy Arruda

Acho que ainda é precoce afirmarmos que a toxina botulínica é uma terapia segura no tratamento de dores musculares, bruxismos, cervicalgias ou cefaléias primárias, como afirmado em algumas publicações.

Desenvolvida inicialmente para tratamento de desordens motoras, o emprego dessa toxina provo- 
ca paralisia muscular, o que tem sido considerada uma forma de terapia para pacientes com dores orofaciais, associadas ou não ao bruxismo.

Muitos estudos têm sido conduzidos com o BOTOX em aplicações de "Trigger Points" miofasciais e cefaléias tensionais, porém não acredito haver evidências suficientes para sua utilização como rotina, no momento. $\mathrm{O}$ custo e alguns problemas nas técnicas de infiltração também devem ser considerados.

Acredito, porém, que seja uma forma de terapia bastante promissora para um futuro próximo, quando se esclarecerem alguns mecanismo centrais de ação dessa droga.

10) No campo das DTMs, algumas dores têm seu curso limitado e o paciente responde bem a vários tratamentos, inclusive placebos. O senhor acha que este falso "sucesso" em alguns tratamentos dificulta a transferência científica em Dor Orofacial, acarretando vários profissionais presos a conceitos ultrapassados? Como resolver este problema? Elcy Arruda

Este é um problema relacionado a todas as áreas de saúde. $\mathrm{Na}$ Medicina, muitas receitas milagrosas de cura tornam-se populares por falta de informação e crendices populares, sendo que apresentaria remissão espontânea de qualquer maneira.

Muitos dos tratamentos odontológicos sugeridos para as dores musculares e articulares demandam períodos longos de acompanhamento, como reabilitações protéticas totais e tratamento ortodôntico. Como afirmado, grande parte dessas dores cessaria de maneira espontânea, mesmo sem a terapia oclusal.

A informação e divulgação de trabalhos bem elaborados e o fortalecimento de um ensino qualificado nas especializações de DTM e Dor Orofacial devem ser os caminhos para a criação de um melhor entendimento de técnicas adequadas de tratamento desses processos.

11) Quais ruídos articulares são considerados como desordens temporomandibulares? Guilherme Janson

Ruídos articulares são bastante comuns em amostras de indivíduos saudáveis, apesar de mais prevalentes em pacientes com dor e disfunção da ATM. Esses ruídos são divididos de maneira didática em estalidos (ou clickings), crepitações e barulhos resultantes de hipertranslação condilar.

Os estalidos são resultado, na maioria dos casos, de desalinhamento entre o côndilo, a eminência e o disco articular, sendo que os momentos de perda e recaptura do disco podem provocar o ruído (deslocamentos do disco articular com redução) (Fig. 1). Já as crepitações são freqüentemente resultado de alterações morfológicas nos componentes da ATM, fruto de degenerações por sobrecarga mecânica ou doenças sistêmicas. É tipicamente relatado pelo paciente como "areia no ouvido". Este tipo de barulho parece ser mais comum em indivíduos idosos, que apresentam algum grau de alteração morfológica da ATM, porém sem a presença obrigatória de dor e/ou disfunção (Fig. 2).

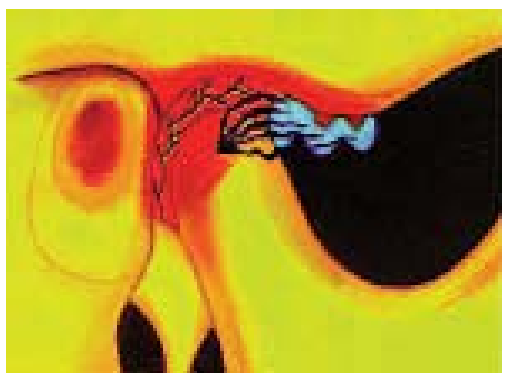

FIGURA 1 - Deslocamento anterior do disco articular.

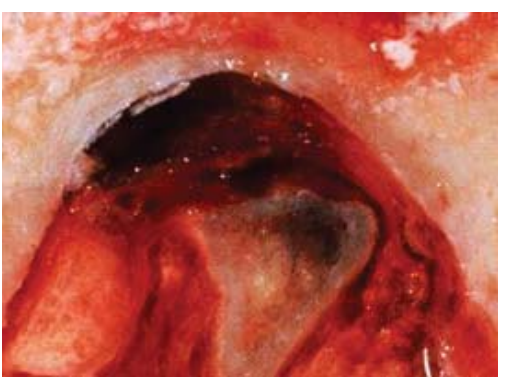

FIGURA 2 - Presença de erosão condilar.

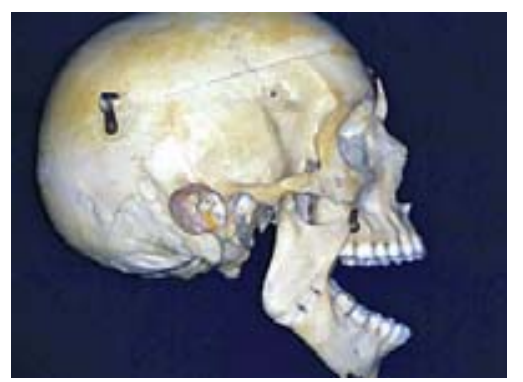

FIGURA 3 - Hipertranslação condilar. 
O barulho por hipertranslação ocorre quando o côndilo mandibular ultrapassa a crista da eminência articular em pacientes com hipermobilidade articular, no final da translação condilar. Normalmente é associado a uma abertura excessiva da boca (Fig. 3).

Apesar desta divisão didática, a identificação do tipo de ruído no exame físico nem sempre é uma tarefa simples, o que dificulta a formação de um diagnóstico correto. Em trabalho realizado por nosso grupo de pesquisa, constatou-se uma baixa concordância para este diagnóstico, mesmo quando realizado por profissionais experientes na área.

Apesar de constituir a queixa principal de muitos pacientes, o tratamento do ruído articular é difícil e de prognóstico duvidoso. Técnicas de reposicionamento anterior da mandíbula, seguido de tratamento ortodôntico ou protético reabilitador, eram usadas no passado, porém com altos índices de recidiva. Algumas técnicas cirúrgicas também são sugeridas para esse fim, porém o caráter invasivo desse procedimento torna sua indicação bastante questionável.

Estudos mostram que a evolução dos estalidos é bastante benigna, sendo que apenas 6 a 7\% dos casos poderão se tornar problemáticos ${ }^{10}$. Além disso, aproximadamente $33 \%$ da população assintomática têm deslocamentos de disco sem problemas de disfunção. Como relatado anteriormente, a presença de um ruído pode ser um indicativo de uma alteração morfológica da ATM, mas não necessariamente vai requerer tratamento.

Dessa forma, não podemos considerar os barulhos da ATM como DTM, sendo que a terapia deve sempre ser voltada para o controle da dor e da disfunção, e não para a eliminação do barulho.

\footnotetext{
12) Qual o mecanismo de ação das placas miorrelaxantes no tratamento das DTMs? Guilherme Janson

Vários mecanismos têm sido sugeridos como responsáveis pela eficácia observada na maioria dos pacientes que utilizam as placas oclusais es-
}

tabilizadoras (ou miorrelaxantes). Deve ficar claro que a utilização das placas deve ser feita após um exame detalhado e diagnóstico correto de cada caso. Ainda, as placas oclusais devem ser utilizadas em conjunto com uma série de modalidades, que incluem fisioterapia, farmacoterapia, aconselhamento, psicoterapia, etc.

Dentre os mecanismos propostos para esta eficácia observada estão o aumento da DVO, o restabelecimento de uma oclusão ideal, alterações na posição condilar e descompressão da ATM, uma alteração na percepção periodontal, relaxamento muscular inicial, alteração cognitiva e placebo.

Tida como uma forma de diagnóstico diferencial no passado devido ao fator oclusal, atualmente sabe-se que esta grande abrangência de efeitos terapêuticos de ação das placas nos leva a questionar a indicação de qualquer tipo de terapia oclusal irreversivel para "complementação"do tratamento, baseado somente no sucesso das mesmas para a diminuição dos sintomas.

Trabalhos de pesquisa em nosso departamento ${ }^{11,12}$ mostraram que mesmo placas não-oclusivas (sem nenhuma alteração na oclusão) podem ser efetivas na diminuição de sintomas, demonstrando todo o poder da alteração cognitiva e efeito placebo desta modalidade de terapia em pacientes com DTM (Fig. 4).

Apesar de não estarem disponíveis trabalhos controlados que comprovem que um mecanismo

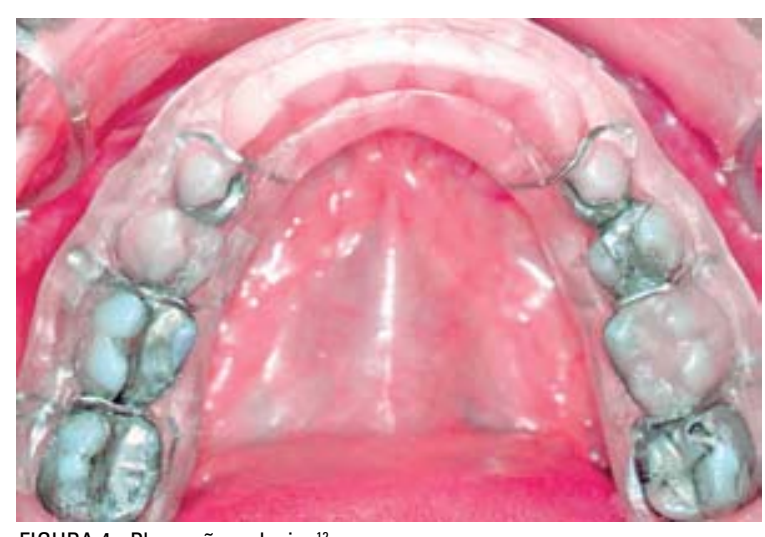

FIGURA 4 - Placa não-oclusiva ${ }^{12}$. 
é mais ou menos importante que outro, o aspecto não-invasivo das placas faz com que a mesma continue sendo uma importante aliada no tratamento de DTMs de origem muscular e articular, em pacientes portadores de hábitos parafuncionais, especificamente bruxismo e apertamento noturno.

13) Existem ocasiões em que se tratam as desordens temporomandibulares com aumento da dimensão vertical? Guilherme Janson

A participação do aumento ou diminuição da DVO como fator etiológico das DTMs tem sido revista nos últimos anos. Historicamente considerada por Costen $^{13}$ como um fator fundamental no desencadeamento de processos de dor e disfunção, alterações na DVO parecem não exercer grande influência no surgimento ou na melhora dos sintomas.

Alguns casos, onde se "restabelece" a DVO por meio de reabilitações orais (RO) completas (por motivos estéticos e de viabilidade protética), pacientes podem relatar uma melhora na dor. Porém, como afirmado anteriormente, muitos desses pacientes poderiam apresentar melhora, independente da terapia, devido ao caráter de remissão espontânea e auto-limitante de algumas condições.

Não podemos nos esquecer que, em casos de aumento da DVO com RO ou troca de dentaduras já desgastadas, por exemplo, há um inegável ganho na estética e auto-estima do paciente, o que pode criar um aspecto psicológico secundário, bastante positivo para a melhora dos sintomas.

No entanto, a instituição de terapias oclusais irreversíveis de aumento da DVO, com o único intuito de tratar as DTMs, não encontra suporte científico em nossos dias.

14) Levando em consideração sua excepcional formação e grande experiência no ensino na área das DTM e Dor Orofacial, como tal área deveria ser desenvolvida nos cursos de Pós-graduação? Eduardo Grossmann
Obrigado por suas palavras, amigo Grossman. Após a consolidação da especialidade e o reconhecimento pelo CFO, acredito que o ensino em nível de Pós-Graduação deva seguir os mesmos passos que outras especialidades criadas também recentemente, como a área dos implantes dentários.

Pesquisas realizadas em nosso país na área de Dores Orofaciais e DTM são realizadas como parte de cursos de Pós-graduação em Estomatologia, Ortodontia e Reabilitação Oral, entre outras. O ensino também é, ainda, fragmentado dentro de disciplinas vinculadas a programas de Mestrado e Doutorado nas áreas já citadas, o que dificulta a formação de docentes e divulgadores de nossa área de maneira acadêmica adequada.

Creio que a formação docente nessa área deva seguir critérios já bem estabelecidos em Programas ou Áreas de Concentração já existentes com sucesso, ou seja, o conhecimento da literatura, o treinamento didático e a experiência com pesquisa são fundamentais para a qualificação de pessoal de nível superior.

A área de DTM e Dores Orofaciais apresenta, ainda, uma particularidade, que é a necessidade de estar em constante contato com outras áreas da saúde, como a Medicina, a Fisioterapia, a Fonoaudiologia e a Psicologia. Dessa maneira, a criação de Cursos de Pós-Graduação, nos moldes dos Mestrados e Doutorados acadêmicos atuais deve estar vinculada à existência destas áreas afins como participantes do Programa.

15) Qual seria o limite entre o tratamento clínico e o cirúrgico no deslocamento do disco sem redução? Eduardo Grossmann

Esta é uma questão que deve ser analisada individualmente, dependendo do grau de dor e disfunção de cada paciente. A maioria dos trabalhos de acompanhamento longitudinal de pacientes com essa condição, sem tratamento, demonstra que há uma tendência de redução da dor e melhora no grau de abertura da boca com o passar do tempo. Sato et al. ${ }^{14}$ e Kurita et al. ${ }^{15}$ demonstraram haver 
entre $30 \%$ e $40 \%$ de remissão dos sintomas em períodos entre 1 e 2 anos.

Estes dados nos alertam em relação à necessidade e à viabilidade de intervenções cirúrgicas num primeiro momento. Os tratamentos conservadores devem ser a primeira opção. Para casos de deslocamento sem redução do disco articular, são utilizadas técnicas de aconselhamento, placas oclusais (estabilizadoras e reposicionadoras, em alguns casos, onde a lesão foi mais recente e há a possibilidade de recaptura temporária do disco articular), fisioterapia (normalmente utilizada para aumento da abertura bucal, em casos crônicos) e farmacoterapia (antiinflamatórios não-esteroidais e relaxantes musculares, para controle de contrações secundárias).

Toda a controvérsia em relação ao tipo de terapia para esses pacientes é devido ao possível risco em manter-se um disco deslocado sem redução em caráter permanente e, dessa maneira, predispor essa articulação a apresentar alterações degenerativas no futuro, como resultado da perda da proteção natural do disco articular. A capacidade regenerativa dos tecidos retrodiscais, com a formação de um pseudo-disco parece suprir essa ausência do disco interposto, na maioria dos pacientes.

Minha experiência (mesmo considerando-se o baixo grau de evidência desse tipo de relato...) em casos desse tipo, é que os pacientes tratados de maneira conservativa conseguem recuperar uma abertura bucal satisfatória, apresentando dor somente em alguns casos, quando submetem a ATM a pressões exageradas, como na mastigação de alimentos mais duros, por exemplo.

Há casos, no entanto, em que a dor se mantém muito forte, a disfunção mandibular não apresenta resposta a nenhuma estratégia de controle, há uma comprovação, por meio de imagens (Fig. 5), de alterações morfológicas da ATM e todo esse quadro interfere com as atividades diárias do paciente. Nessas condições, acho que uma consulta cirúrgica deva estar indicada, na tentativa de se restabelecer condições ideais para a ATM dolorida.

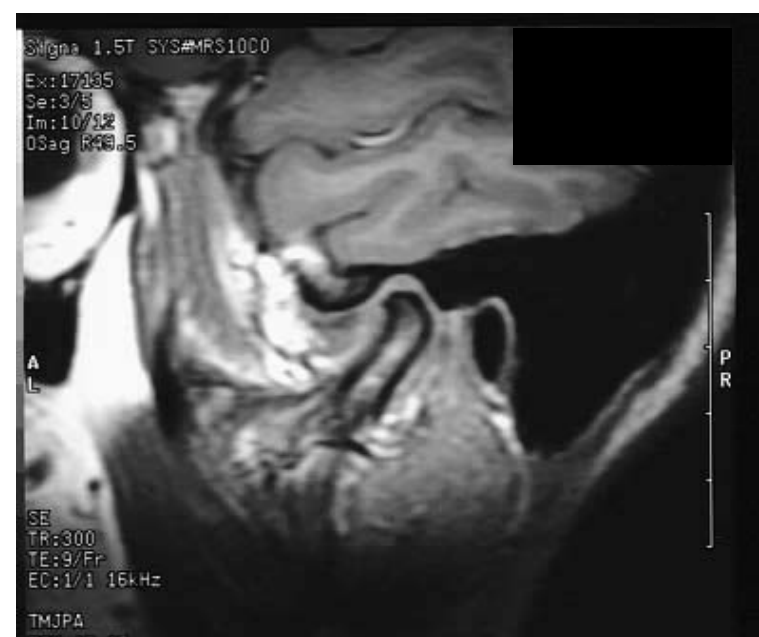

FIGURA 5 - Ressonância Magnética, onde se observa deslocamento do disco articular, associado a alterações degenerativas.

Não se pode esquecer, no entanto, que casos crônicos apresentam processos de sensitização neuronal periférica e central, que podem comprometer o resultado de alívio da dor, almejado por meio dos procedimentos cirúrgicos.

16) Dentre as terapêuticas empregadas no momento, qual(is) a(s) mais eficaz(es) em se tratando de pacientes portadores de um quadro de dor e disfunção miofacial ? Guilherme Janson

O tratamento da dor miofacial é baseado na eliminação dos pontos gatilhos, na redução ou eliminação de condições predisponentes e dos fatores que podem levar à recidiva. $\mathrm{O}$ sucesso do tratamento ocorre com mais efetividade quando uma abordagem multidisciplinar é usada.

A meta do tratamento em curto prazo é restaurar o comprimento normal do músculo, melhorar a postura e obter uma total movimentação das articulações. $\mathrm{O}$ tratamento em longo prazo envolve um programa regular de alongamento muscular, manutenção da correta postura e programa de exercícios de musculação.

Dentre as técnicas de tratamento das dores miofasciais estão o aconselhamento e alteração comportamental e postural, eliminação de hábi- 
tos deletérios, massagens, compressão dos pontos gatilhos, crioterapia e alongamento muscular e infiltração desses pontos.

Fazem parte do aconselhamento, medidas tais como:

- manter uma boa postura, descansar os músculos mastigatórios, através da limitação voluntária das atividades - evitar alimentos consistentes e se resguardar de atividades que causam dor nos músculos mastigatórios, como mascar chicletes, e consultas odontológicas prolongadas sem descansos;

- estar ciente e eliminar os hábitos parafuncionais.

- aplicar compressas de calor ou frio nas áreas da musculatura doloridas.

- evitar hábitos de dormir que forcem os músculos e as articulações. Não dormir de bruços, e se dormir de lado, manter o pescoço e a cabeça alinhados.

- evitar cafeína, pois esta estimula os músculos a contraírem-se, tornando-os mais tensos.

Massagens têm sido sugeridas para aliviar a dor, aumentar o fluxo sangüíneo para os músculos e elevar os níveis de endorfina plasmática. Esses níveis mantêm-se elevados por aproximadamente 90 minutos após a massagem e isto pode ser responsável pelo alívio da dor, relaxamento e bemestar relatado pelos pacientes.

Técnicas de compressão também têm sido utilizadas e sugeridas nos clássicos tratados de Travell e Simons, e mais atualmente em publicações de Mense e Simons. O ponto gatilho deve ser pressionado até o limite da dor e mantido dessa maneira por um minuto e, então, liberado.

Já a técnica de crioterapia e alongamento passivo parece ser efetiva para a maioria dos casos. O congelamento da pele e excessiva varredura devem ser evitados, porque estes podem diminuir a temperatura do músculo esquelético, tendendo a agravar os pontos gatilhos.

Infiltração dos pontos gatilhos têm se mostrado eficiente na redução da dor, no aumento do limite de movimento, aumento da tolerância de exercícios e aumento na circulação dos músculos. Geralmente é realizada quando não houve respostas efetivas com as técnicas já descritas. Raramente os pacientes com dor miofacial crônica obtêm alívio permanente com uma única infiltração. Uma série de injeções, coordenadas com exercícios dos músculos afetados, produzem um melhor resultado em longo prazo.

O alívio da dor pode variar da duração do anestésico a vários meses, dependendo da cronicidade e severidade dos pontos gatilhos, e o grau de redução dos fatores perpetuantes. Desde que a perturbação mecânica dos pontos gatilhos pela agulha é um fator crítico no alívio, a precisão no agulhamento parece ser o principal fator da inativação dos mesmos. A utilização de anestésico local (procaína $0,5 \%$, sem vasoconstritor) tem sido mais freqüente em infiltrações deste tipo. Porém, há vários trabalhos, onde foi utilizado o agulhamento seco ou com outras substâncias, tais como o soro fisiológico ou a enzima botulínica, sem diferenças significativas, quando comparada ao agulhamento com o anestésico.

Apesar de não haver estudos controlados demonstrando a eficácia de todas estas técnicas, as mesmas têm sido utilizadas com relatos de sucesso por vários autores. Como regra geral, da mesma maneira que para qualquer condição, recomenda-se dar preferência para as modalidades menos invasivas.

17) $O$ que podemos esperar quanto ao emprego de células tronco como terapêutica nas Dores Orofaciais ? Eduardo Grossmann

Bastante estudada atualmente, a utilização de tecidos a partir de células-tronco parece ter um futuro promissor dentro da Medicina e, provavelmente, da Odontologia. A criação de tecidos dentários, periodontais e ósseos, a partir de células indiferenciadas, abre perspectivas de terapia odontológica até então inviáveis e impossíveis.

O campo das dores orofaciais também deve 
se beneficiar desse tipo de terapia, com a possibilidade de crescimento tecidual em várias áreas degeneradas. Processos de dores crônicas e de alterações neuronais centrais, como a expressão de receptores NMDA, e atividade de neurotransmissores excitatórios centrais e periféricos continuarão, no entanto, a constituir desafios na batalha incessante contra as dores orofaciais.

\section{REFERÊNCIAS}

1. CONTI, P. C. R.: MIRANDA, J. E. S.: ORNELAS, F. Ruídos articulares e sinais de disfunção temporomandibular: um estudo comparativo por meio da palpação manual e vibratografia computadorizada da ATM. Rev Pesq Odontol Bras, [S.I..], v. 14 n. 4, p. 367-371, out./dez.2000

2. CONTI, P. C. R.; GONÇALVES, L. F.; KANO, S. C.; CONTI, A C. C. F.; CONTI, J. V. Avaliação da prevalência das dores de cabeça primárias e seu relacionamento com sintomas de desordens temporomandibulares no Campus da USP, na Cidade de Bauru/SP. R Dental Press Ortod Ortop Facial, Maringá, v. 8 , n. 2, p. 49-56, mar./abr. 2003

3. WINOCUR, E. et al. Drugs and bruxism: a critical review. J Orofac Pain, Carol Stream, v. 17, no. 2, p. 99-111, Spring, 2003.

4. LOBBEZOO, F.; VAN DENDEREN, R. J.; VERHEIJ, J. G.; NAEIJE, M. Reports of SSRI-associated bruxism in the family physician's office. J Orofac Pain, Carol Stream, v. 15, no. 4, Fall, p. 340-6, 2001.

5. GERBER, P. E.; LYND, L. D. Selective serotonin-reuptake inhibitor-induced movement disorders. Ann Pharmacother Cincinnati, v. 32, no. 6, p. 692-8, Jun. 1998.

6. FORSSELL, H.; KALSO, E.; KOSKELA, P.; VEHMANEN, R.; PUUKKA, P.; ALANEN, P. Occlusal treatments in temporomandibular disorders: a qualitative systematic review of randomized controlled trials. Pain, Amsterdam, v. 83, no. 3, p. 549-60 Dec.1999.

7. TEIXEIRA, M. J. Dor: contexto interdisciplinar. Ed. Santos, 2002 cap. 31

8. TEIXEIRA, M. J.; PIMENTA, C. A. M. Epidemiologia da dor. In: TEIXEIRA, M. J. Dor: conceitos gerais. São Paulo: Limay, 1995. p. 57-61

9. MCNAMARA, J. A.; SELIGMAN, D. A.; OKESON, J. P. Occlusion, orthodontic treatment and temporomandibular disorders: a review. J Orofac Pain, Carol Stream, v. 9, p. 73-90, 1995.

10. OKESON, J. P.; HAYES, D. K. Long-term results of treatment for temporomandibular disorders: an evaluation by patients. J Am Dent Assoc, Chicago, v. 112, no. 4, p. 473-8, Apr. 1986.

11. GUEDES, J. R. Avaliação do efeito placebo em pacientes portadores de disfunção craniomandibular, através do uso de placas miorrelaxantes convencionais e placas não oclusivas. 1994. Dissertação (Mestrado)-Faculdade de Odontologia de Bauru, Universidade de São Paulo, São Paulo,1994.

12. SANTOS, C. N. Avaliação da eficácia de placas estabilizado ras com padrão de guia balanceada bilateral no controle dos desarranjos internos da ATM. 2003. Tese (Doutorado)-Faculdade de Odontologia de Bauru, Universidade de São Paulo, São Paulo, 2003

\section{Antonio Sérgio Guimarães}

- Prof. Dr. responsável pela área de Odontologia do Ambulatório de DTM e Dor Orofacial - Hospital São Paulo - UNIFESP/EPM:

- Coordenador do Curso de Mestrado - área de concentração DTM/Dor Orofacial, Centro de Pós-Graduação em Odontologia SL Mandic.

\section{Eduardo Grossmann}

- Doutor e Mestre em Odontologia com temática voltada para Dor Orofacial e DTM; Especialista em DTM e Dor Orofacial.

- Prof. do Curso de Pós-graduação em Dor da Faculdade de Medicina da UFRGS

- Consultor na área de DTM e DORF do Serviço de Dor do Hospital de Clínicas de Porto Alegre.

- Diretor do Núcleo de Dor Orofacial e DTM da Sociedade Gaúcha para o Estudo da Dor.

- Diretor do Centro de Dor e Deformidade Orofacial CENDDOR, Porto Alegre/RS

\section{Eleutério Araújo Martins}

- Prof. Titular Faculdade de Odontologia UFRGS.

- Doutor em Odontologia UFRGS.

- Livre Docente UFRGS.

- Pós Doutorado - University of Michigan, USA (1960-1961).

- Coordenador do Curso de Especialização em DTM e Dor Orofacial, ABO-RS.

- Presidente AGEDOF ( Associação Gaúcha para o Estudo e Pesquisa da Dor Orofacial).

\section{Elcy Arruda}

- Especialista em Ortodontia e Ortopedia Facial pela AMOABO - Maringá.

- Especialista em Disfunção Temporomandibular e Dor Orofacial.

- Mestrando em Odontologia pela PUCPR.

\section{Guilherme Janson}

- Professor Associado do Departamento de Ortodontia da Faculdade de Odontologia de Bauru - USP.

- Coordenador do curso de Mestrado em Ortodontia da FOB-USP.

- Pós-doutorado na Universidade de Toronto - Canadá

13. COSTEN, J. B. A syndrome of ear and sinus symptoms dependent upon distuberd functions of TMJ. Ann Otol, St. Louis, v. 43, no. 1, p. 1-15, Mar. 1934.

14. SATO, $\mathrm{S}$. et al. The natural course of nonreducing disc displacement of the TMJ: relationship of clinical findings at initial visit to outcome after 12 months without treatment. J Orofac Pain, Carol Stream, v. 11, no. 4, p. 315-20, Fall, 1997.

15. KURITA, K. et al. Natural course of untreated symptomatic temporomandibular joint disc displacement without reduction. J Dent Res, Chicago, v. 77, no. 2, p. 361-65, 1998. 